\title{
High precision frequency measurement of terahertz waves using optical combs from a Mach-Zehnder-modulator-based flat comb generator
}

\author{
Isao Morohashi, ${ }^{1, *}$ Ikufumi Katayama, ${ }^{2}$ Mayu Kirigaya, ${ }^{2}$ Yoshinisa Irimajiri, ${ }^{1}$ \\ Norihiko Sekine, ${ }^{1}$ and Iwao Hosako ${ }^{1}$ \\ ${ }^{1}$ National Institute of Information and Communications Technology, Koganei, Tokyo 184-8795, Japan \\ ${ }^{2}$ Department of Physics, Graduate School of Engineering, Yokohama National University, Yokohama 240-8501, Japan \\ *Corresponding author: morohashi@nict.go.jp
}

Received 30 October 2018; accepted 12 December 2018; posted 18 December 2018 (Doc. ID 349579); published 16 January 2019

Using a frequency-tunable optical comb generated from a Mach-Zehnder-modulator-based flat comb generator (MZFCG) and a nonlinear optical fiber, we demonstrated a frequency measurement of continuous terahertz wave sources with the frequency of 0.1 and $0.6 \mathrm{THz}$ by an electro-optic sampling method. We clearly observed beat signals between the terahertz source and the optical two-tone extracted from the optical comb, allowing us to determine the absolute frequency. Owing to the wide comb spacing of the MZ-FCG, this method has a high potential for the high-speed measurement of the frequency of terahertz wave sources. (c) 2019 Optical Society of America

https://doi.org/10.1364/OL.44.000487

The terahertz frequency region attracts much attention because it offers complementary tools for x-ray imaging [1], can be a carrier wave for ultrahigh bandwidth wireless communication [2], and is useful for highly sensitive gas spectroscopy for environmental monitoring $[3,4]$. For these applications, it is of increasing importance to precisely and easily monitor the absolute frequency of terahertz light sources with high sensitivity and speed. However, measurement methods for determining the terahertz intensity require complicated setups for cooling down the detector, since the energy of a terahertz photon is much lower than room temperature, and the thermal noise in the detector degrades the sensitivity $[5,6]$.

In the last few decades, a new detection method for terahertz waves has emerged from ultrafast laser technology, in which the terahertz electric field is measured using the electro-optic (EO) effect in semiconductor crystals [7-9]. Since this method measures the coherent part (electric field) of electromagnetic waves, it is unnecessary to operate at cryogenic temperatures. Furthermore, since the EO effect arises from the difference frequency generation process, the carrier envelope phase or frequency offset does not affect the measurement, and the observed beat frequency depends only on the index of frequency comb and the comb spacing [7]. Therefore, if the sensitivity of the electric field detection becomes good enough, the EO sampling may be a promising method for terahertz applications.

Recently, the detection of terahertz frequency using modelocked solid-state or fiber lasers has been demonstrated, and promising capabilities in frequency metrology have been revealed [7-11]. In this method, it is impossible to measure the frequency of terahertz waves that have a spectral width exceeding half the repetition frequency (typically tens of megahertz in mode-locked lasers), so that a higher repetition frequency is desirable. However, it is difficult to realize the comb spacing greater than several gigahertz due to the difficulty of reducing the cavity length for the mode-locking [12]. If the repetition frequency becomes higher, the mode intensity of the optical comb increases, and the detection bandwidth can be significantly wider than in the conventional mode-locked laser-based technologies. Here we propose that the MachZehnder-modulator (MZM)-based flat comb generator (MZFCG) offers a unique advantage over the mode-locked laser based frequency measurements, as the comb spacing can be easily tuned $[13,14]$.

Figure 1 shows a schematic of our scheme for the terahertz frequency measurement. This system is based on an optical heterodyne via an EO sampling using optical combs. An EO crystal acts as an optical modulator driven by terahertz waves. When a terahertz wave with a frequency of $f_{\mathrm{THz}}$ is irradiated on the EO crystal, a change in the refractive index is induced that is proportional to the electric field strength of the terahertz wave. This temporally periodic index change gives rise to a phase modulation of the optical probe collinearly introduced into the $\mathrm{EO}$ crystal, generating modulation sidebands on both sides of the original comb. Although the intensity profile of the sidebands follows the Bessel function, only the first-order term is taken in account, since the modulation index induced by the terahertz wave is small. 


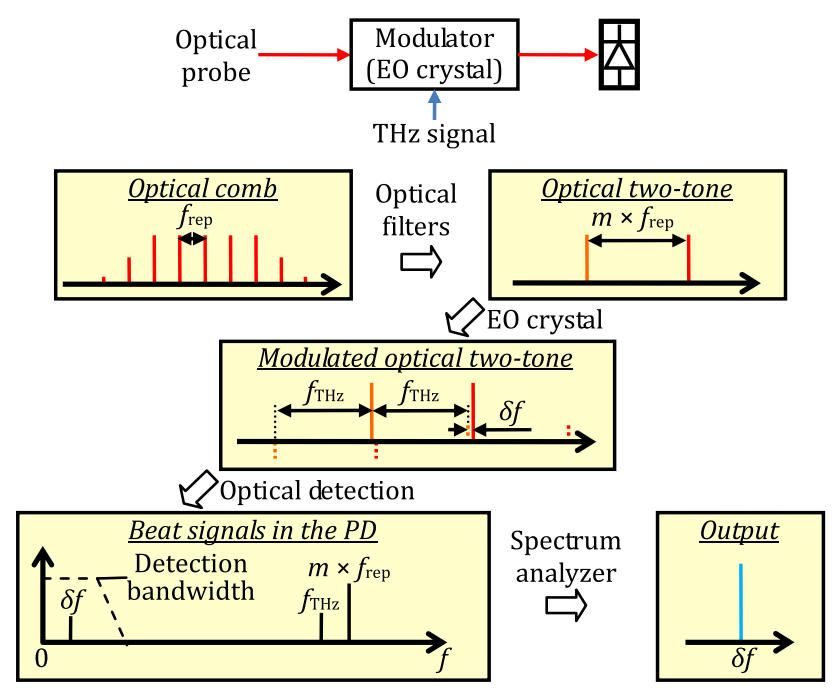

Fig. 1. Schematic of terahertz frequency measurement using the EO sampling method.

To facilitate the terahertz detection, we extracted an optical two-tone from the optical comb. Owing to the wide comb spacing $f_{\text {rep }}$ of the optical comb, we could easily extract the desired comb modes by using optical bandpass filters. The spacing of the optical two-tone is set to $m \times f_{\text {rep }}$ ( $m$ is an integer), which is closest to the terahertz source. In the EO crystal driven with the terahertz wave, modulation sidebands are created with the frequency separation of $f_{\mathrm{THz}}$. By detecting the modulated optical probe using a photodetector (PD), beat notes are generated in the RF region. Owing to the detection bandwidth, only the lowest frequency component of the beat notes could be observed. By measuring the beat frequency $\delta f$, the terahertz frequency $f_{\mathrm{THz}}$ can be precisely determined:

$$
f_{\mathrm{THz}}=m f_{\text {rep }} \pm \delta f \text {. }
$$

In this scheme, the accuracy and stability of the frequency are determined by those of $f_{\text {rep }}$, so that $f_{\text {rep }}$ has to be highly stabilized for a high precision frequency measurement. Furthermore, stabilization of the carrier envelope offset and compensation of the phase dispersion of the optical comb are not required, because the frequency offset of the optical comb is canceled out during downconversion and only two comb modes, respectively.

Figure 2 shows the experimental setup for terahertz frequency measurement [15]. As the terahertz source, a Gunn oscillator combined with a sixfold frequency multiplier was used. The Gunn oscillator operated in a $0.1 \mathrm{THz}$ band with an output power of $20 \mathrm{~mW}$, and then the frequency of the multiplier was in a $0.6 \mathrm{THz}$ band with an output power of $0.3 \mathrm{~mW}$. For EO sampling, a (110)-oriented 1-mm-thick ZnTe crystal was used. Terahertz waves emitted by the terahertz source were focused on the EO crystal using a pair of off-axis parabolic mirrors with a focal length of $50 \mathrm{~mm}$. The polarization of the terahertz waves was aligned with the $\langle 001\rangle$-direction, inducing an index change in the $\langle-110\rangle$ direction. An optical probe beam was incident on the EO crystal, collinear with the terahertz waves. The polarization of the optical probe was set at an angle of $45 \mathrm{deg}$ with the $\langle 001\rangle$ direction. In this situation, there are two propagation modes, $\langle 001\rangle$ and $\langle-110\rangle$.

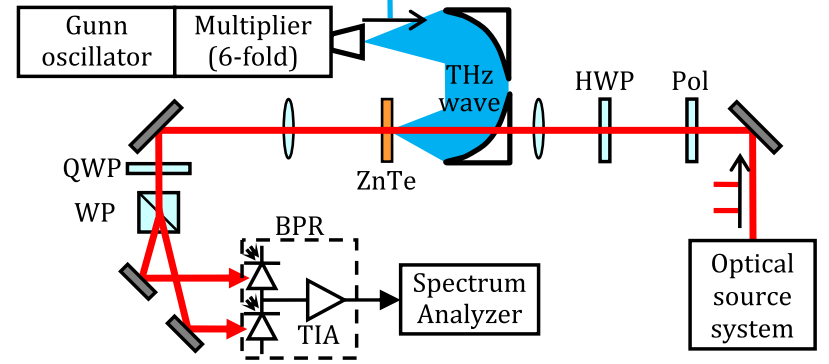

Fig. 2. Experimental setup for the frequency measurement of terahertz waves. Pol, polarizer; HWP, half-wave plate; QWP, quarterwave plate; WP, Wollaston prism; BPR, balanced photoreceiver; TIA, transimpedance amplifier.

The $\langle-110\rangle$ mode is modulated by the terahertz waves, and the other mode is unmodulated. As a result, a temporal change in the polarization of the optical probe is induced at the output of the EO crystal. This polarization change was analyzed by a combination of a quarter-wave plate and Wollaston prism, and then detected by a balanced photoreceiver (BPR) (Newport 2017, $125 \mathrm{kHz}$ bandwidth, conversion gain $1 \times 10^{6} \mathrm{~V} / \mathrm{W}$ ). The frequency difference between the terahertz waves and the optical two-tone was output as beat notes. Spectral monitoring and frequency measurement of the beat notes were performed using an RF spectrum analyzer.

The optical source used in this experiment mainly consists of the MZ-FCG. The MZ-FCG adopts the optical comb generation method based on optical modulation and converts a continuous wave $(\mathrm{cw})$ light into an ultraflat optical comb with equal spacing between modes. Details of the MZ-FCG on the operation principle and configuration are described in Refs. [13,14]. In the MZ-FCG, a dual-drive-type MZM is driven by a microwave signal under a specific condition (flat spectrum condition), and a cw light is fed to it as a seed light. The comb spacing is determined by the frequency of the microwave signal, and the center wavelength is equal to that of the seed light, so that the center wavelength and the comb spacing can be controlled independently. An important feature of this source is that the comb spacing $f_{\text {rep }}$ can be widely tuned in the range from 6 to $18 \mathrm{GHz}$ with a millihertz precision by changing the frequency of the microwave signal [14], which leads to the precise tuning of the comb spacing. These features are completely different from mode-locked lasers. Figure 3(a) shows the configuration of the optical comb source. The MZM was driven by a variable-frequency microwave signal with a power of $16 \mathrm{dBm}$ generated by a signal generator (SG), and a cw light output from a laser diode (LD) with a wavelength of $1550.8 \mathrm{~nm}$ and a power of $10 \mathrm{dBm}$ was used for the seed light. By satisfying the flat spectrum condition (defined by the amplitude difference and the bias voltage applied to the two arms of the MZM), flat optical combs are generated, as shown in Fig. 3(b). The $10 \mathrm{~dB}$ bandwidth was $290 \mathrm{GHz}$ under the condition of $f_{\text {rep }}=10 \mathrm{GHz}$.

To measure the frequency bands of $0.6 \mathrm{THz}$ or higher, a highly nonlinear dispersion shifted fiber (HNL-DSF) was used to broaden the bandwidth of the optical comb. For efficient spectral broadening, prior to inputting the comb into the HNL-DSF, the optical combs were converted into pulse trains by a chirp compensation using an 1100-m-long single-mode 

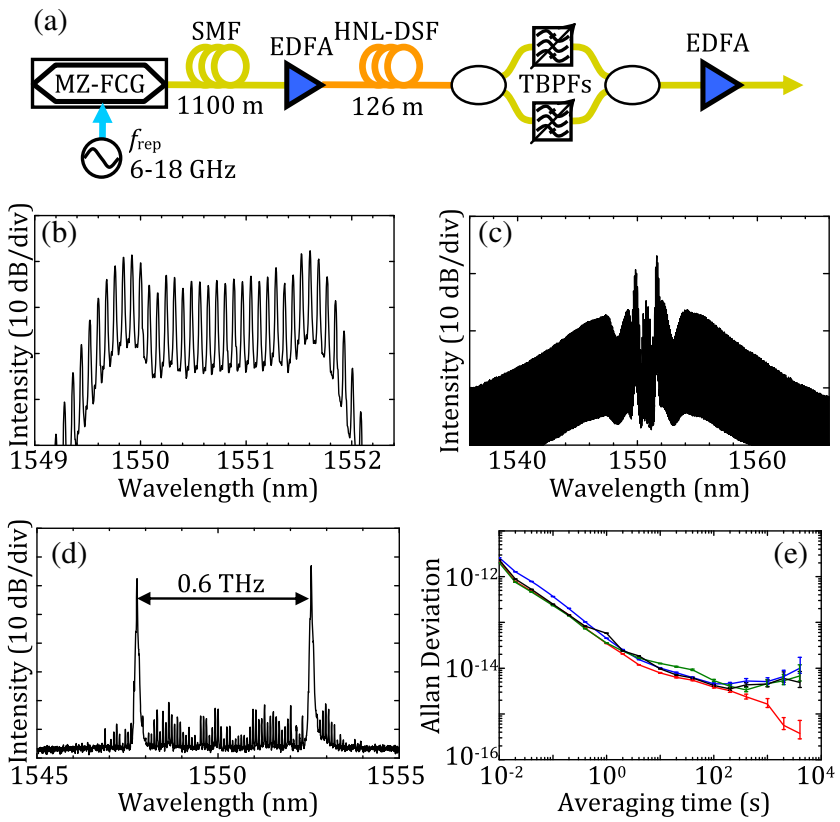

Fig. 3. (a) Configuration of the optical two-tone source. Spectra of (b) a $10 \mathrm{GHz}$-spaced optical comb from the MZ-FCG, (c) a broadband optical comb generated by the HNL-DSF, and (d) an optical two-tone with a frequency spacing of $0.6 \mathrm{THz}$. (e) Allan deviation. Red, the driving signal of the MZ-FCG; blue, the optical comb generated by MZ-FCG; green, the broadband comb; black, a two-tone signal with a spacing of $0.1 \mathrm{THz}$.

fiber (SMF) and amplified to $25 \mathrm{dBm}$ in average by an erbiumdoped fiber amplifier (EDFA).

Figure 3(c) shows the spectrum of a broadband optical comb. The $10 \mathrm{~dB}$ bandwidth of the broadband optical comb was about $2.5 \mathrm{THz}$. It allows one to measure terahertz frequency in the high frequency band by using the broadband optical comb. To create optical two-tones from the optical combs, a pair of optical tunable bandpass filters (TBPFs) with a minimum pass bandwidth of $6 \mathrm{GHz}$ (Yenista, XTM-50 Standard) was used. Figure 3(d) shows the spectrum of an optical two-tone as an example. Two modes were successfully extracted with signal-to-noise ratios (SNRs) of greater than $30 \mathrm{~dB}$. Because the TBPFs can continuously tune both the center wavelength and the bandwidth independently of each other, the spacing of the optical two-tone, $m$, can be set to an arbitrary value.

As mentioned above, the frequency stability of the optical combs is important for high precision in frequency measurement, so that the frequency stability of the optical comb was evaluated. Figure 3(e) shows the Allan deviation of the optical combs, the broadband comb, and an optical two-tone with a spacing of $0.1 \mathrm{THz}$. For comparison, the Allan deviation of the microwave signal is also shown in the figure. Since the frequency range of our equipment for the Allan deviation measurement (Microsemi Corp., 3210A) was up to $30 \mathrm{MHz}$, $10 \mathrm{GHz}$ signals were measured with downconverting to $10 \mathrm{MHz}$ using a mixer with another microwave signal for the LO. By comparing the Allan deviation of the optical combs and the $0.1 \mathrm{THz}$ signal to that of the microwave signal, the degree of degradation in frequency stability from
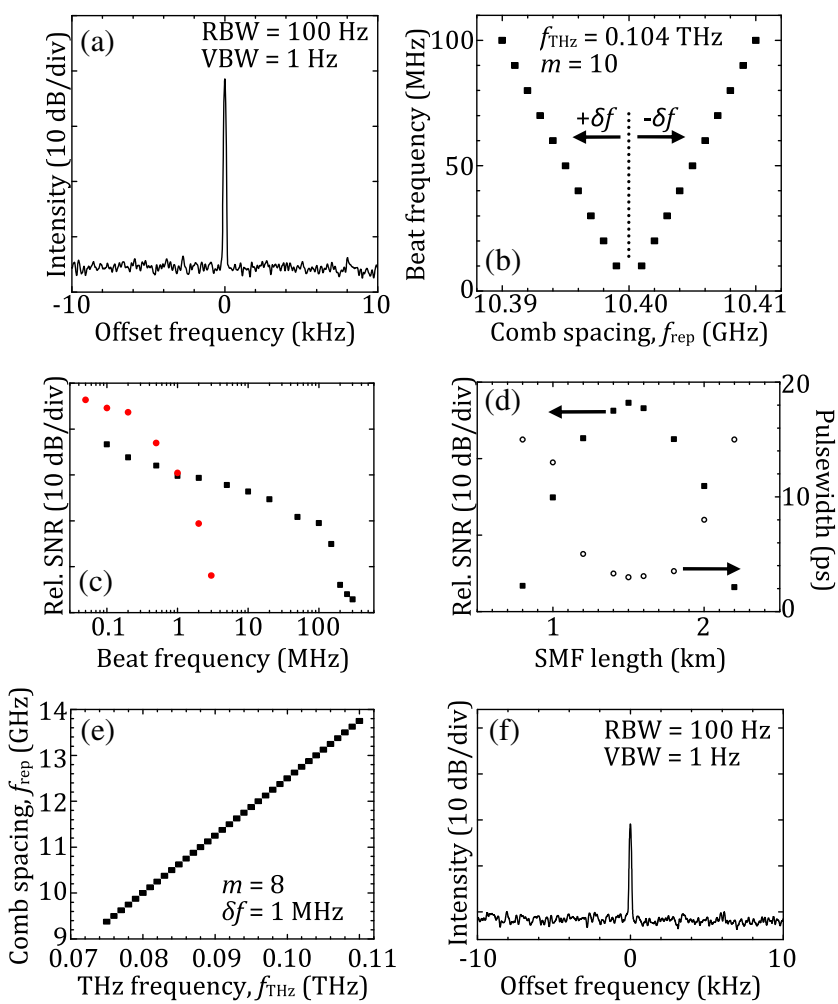

Fig. 4. (a) Spectrum of a $0.1 \mathrm{THz}$ signal. (b) Variation of the beat frequency with the comb spacing. (c) The detection bandwidth of this system. In this figure, two BPRs (red, Newport 2017; black, Newport 1817) were compared. (d) The change in the SNR of $0.1 \mathrm{THz}$ (closed squares) and the pulse width (open circles signal) as a function of the SMF length. (e) Wideband frequency measurement by changing $f_{\text {rep }}$. (f) The spectrum of the $0.6 \mathrm{THz}$ band signal.

the microwave signal caused by our optical source can be evaluated. It can be clearly seen that the frequency stability of the optical two-tone is determined by the microwave signal. The degradation of the frequency stability observed with averaging times over $200 \mathrm{~s}$ is due to instability in the fibers (the SMF and the HNL-DSF). The frequency stability of the optical two-tone with an averaging time of $1 \mathrm{~s}$ was $5.8 \times 10^{-14}$. Since the actual frequency stability of the optical two-tone is multiplied by the magnification factor, this result means that the frequency instability in frequency measurement is of the order of tens of megahertz for the $0.1 \mathrm{THz}$ band. Thus, the MZ-FCG makes it possible to measure terahertz frequencies precisely.

By using the MZ-FCG, a precise frequency measurement was performed. In the case of detection of $0.1 \mathrm{THz}$ band signals, only the Gunn oscillator was used as the terahertz source, and the oscillation frequency $\left(f_{\mathrm{THz}}\right)$ was set to $0.104 \mathrm{THz}$. The spacing of the optical two-tone was set to 10 modes $(m=10)$. Figure 4(a) shows the spectra of a beat signal. When $f_{\text {rep }}$ was set to $10.399990 \mathrm{GHz}$, a beat signal appeared at $100 \mathrm{kHz}(\delta f)$, which was exactly consistent with that calculated using Eq. (1). No frequency drift of the beat signal was observed in a longterm measurement. The linewidth of the beat signal was narrower than $1 \mathrm{~Hz}$, which is less than the minimum frequency resolution of the spectrum analyzer. This result is also consistent with that expected from the Allan deviation. The intensity of the beat signal is maximized when the powers of the two lines 
are balanced due to the presence of the input limit of the BPR. Thus, optical combs with high flatness are suitable for simplifying the frequency measurement system. The SNR was $35 \mathrm{~dB}$ with a resolution bandwidth (RBW) of $100 \mathrm{~Hz}$ and video bandwidth (VBW) of $1 \mathrm{~Hz}$. The noise characteristics of the beat signal are dominated by those of the seed LD, and no increment in the noise caused by the MZ-FCG (modulation and bias noises) was observed. Equation (1) involves ambiguity due to the sign of the beat signal $( \pm \delta f)$. Although two beat signals may appear in the same frequency range, these can be easily distinguished by changing the $f_{\text {rep }}$. Figure 4(b) shows $\delta f$ as a function of $f_{\text {rep. }}$. When $\delta f>0$, the beat frequency has a negative slope as $f_{\text {rep }}$ increases, and it has a positive slope when $\delta f<0$. Therefore, the sign of $\delta f$ can be determined by monitoring the direction of change of $\delta f$ with a change in $f_{\text {rep }}$.

Since $\delta f$ needs to be less than the detection bandwidth, it is an important factor in this system. In our scheme, due to the use of optical two-tone, the detection bandwidth is not limited to half the repetition rate $\left(f_{\text {rep }} / 2\right)$, so that in principle, a wideband operation can be achieved. However, in practice, the detection bandwidth is limited by the optical detection bandwidth. Figure 4(c) shows the detection bandwidths in this system. In this experiment, $f_{\mathrm{THz}}$ was varied, while $f_{\text {rep }}$ and $m$ were fixed. For comparison, another BPR (Newport 1817, $80 \mathrm{MHz}$ bandwidth and a conversion gain of $5 \times 10^{4} \mathrm{~V} / \mathrm{W}$ ) was also used. The result shown in Fig. 4(c) clearly shows that the detection bandwidth and the SNR are mainly determined by the bandwidth and the conversion gain of the BPR.

Although our scheme demonstrated here can be used without TBPFs, the frequency chirp of the optical comb significantly affects the detection efficiency in that case. We investigated the impact of the frequency chirp of the optical comb without TBPFs. Figure 4(d) shows the change in the SNR of terahertz detection as a function of the SMF length (frequency chirp of the optical comb). In the figure, the pulse width of the optical comb obtained at each SMF length was also plotted as an indicator of the chirp state. In this experiment, an optical comb output from the MZ-FCG without using the HNL-DSF was used for EO sampling. The SNR was maximized at an SMF length of $1500 \mathrm{~m}$, which coincides with the shortest pulse width. The result shows that the frequency chirp of the optical comb significantly affects the detection efficiency, and the chirp-free optical combs maximize the detection efficiency. This is due to the destructive interference between the comb modes during the downconversion to the RF region caused by the chirp. The optical two-tone eliminates the chirp of the optical comb, so that the use of the optical two-tone is an effective way to avoid the chirp-induced degradation of detection efficiency, especially if it is difficult to completely compensate for the frequency chirp.

Although the detection bandwidth is limited by that of the $\mathrm{BPR}$, as discussed above, the tunability in $f_{\text {rep }}$ enables a wideband frequency measurement, as shown in Fig. 4(e). In this experiment, $f_{\mathrm{THz}}$ in the range from 0.075 to $0.110 \mathrm{THz}$ was measured by tuning $f_{\text {rep }}$ until $\delta f=1 \mathrm{MHz}$ was obtained at $m=8$, and the resulting $f_{\text {rep }}$ was plotted as a function of $f_{\mathrm{THz}}$. A clear linearity between these two frequencies demonstrates the wideband and precise frequency measurement, which is expressed by Eq. (1).

By combining two tunings in both $f_{\text {rep }}$ (precise tuning) and $m$ (coarse tuning), broadband frequency measurement up to the full bandwidth of the optical comb is possible, regardless of the phase relationship between the comb modes. To demonstrate this feature, a frequency measurement of a $0.6 \mathrm{THz}$ band signal was also performed. When $m$ and $f_{\text {rep }}$ were set to 62 and $10.064514520 \mathrm{GHz}$, respectively, a beat signal was observed at $100 \mathrm{kHz}$. Figure $4(\mathrm{f})$ shows the spectrum of the beat signal. As in the case of a $100 \mathrm{GHz}$ band signal detection, the frequency of the beat signal was exactly equal to that from Eq. (1). The SNR was $20 \mathrm{~dB}$ at RBW of $100 \mathrm{~Hz}$ and VBW of $1 \mathrm{~Hz}$. The linewidth of the beat signal was narrower than the minimum resolution of the spectrum analyzer. These results indicate that the MZ-FCG can be a promising method for detecting absolute frequency without requiring a cryogenic system.

In conclusion, we demonstrated a precise frequency measurement of 0.1 and $0.6 \mathrm{THz}$ bands using the MZ-FCG for precision spectroscopy in the terahertz range. By extracting two comb modes from the optical comb, we could realize the terahertz frequency measurement at room temperature. Since the frequency of the comb mode spacing in the MZFCG can be widely and easily tuned from 6 to $18 \mathrm{GHz}$, this technique could be applied to a wide range of different terahertz sources with different characteristics. The results demonstrate the promising capability of MZ-FCG for terahertz applications.

Funding. Strategic Information and Communications R\&D Promotion Programme of Ministry of Internal Affairs and Communications (MIC) (145003103); Japan Society for the Promotion of Science (JSPS) (JP17K05092).

\section{REFERENCES}

1. B. Ferguson and X.-C. Zhang, Nat. Mater. 1, 26 (2002).

2. A. Kanno, K. Inagaki, I. Morohashi, T. Sakamoto, T. Kuri, I. Hosako, T. Kawanishi, Y. Yoshida, and K. Kitayama, Opt. Express 19, B56 (2011).

3. M. van Exter, C. Fattinger, and D. Grischkowsky, Opt. Lett. 14, 1128 (1989).

4. R. Guo, K. Akiyama, H. Minamide, and H. Ito, Appl. Phys. Lett. 90, 121127 (2007).

5. S. Bartalini, L. Consolino, P. Cancio, P. De Natale, P. Bartolini, A Taschin, M. De Pas, H. Beere, D. Ritchie, M. S. Vitiello, and R. Torre, Phys. Rev. X 4, 021006 (2014).

6. L. Consolino, A. Taschin, P. Bartolini, S. Bartalini, P. Cancio, A. Tredicucci, H. E. Beere, D. A. Ritchie, R. Torre, M. S. Vitiello, and P. De Natale, Nat. Commun. 3, 1040 (2012).

7. S. Barbieri, P. Gellie, G. Santarelli, L. Ding, W. Maineult, C. Sirtori, R Colombelli, H. Beere, and D. Ritchie, Nat. Photon. 4, 636 (2010).

8. M. Ravaro, C. Manquest, C. Sirtori, S. Barbieri, G. Santarelli, K. Blary, J. F. Lampin, S. P. Khanna, and E. H. Linfield, Opt. Lett. 36, 3969 (2011).

9. S. Yokoyama, R. Nakamura, M. Nose, T. Araki, and T. Yasui, Opt. Express 16, 13052 (2008).

10. T. Yasui, K. Hayashi, R. Ichikawa, H. Cahyadi, Y. D. Hsieh, Y. Mizutani, H. Yamamoto, T. Iwata, H. Inaba, and K. Minoshima, Opt. Express 23, 11367 (2015).

11. T. Yasui, S. Yokoyama, H. Inaba, K. Minoshima, T. Nagatsuma, and T. Araki, J. Sel. Top. Quantum Electron. 17, 191 (2011).

12. A. Bartels, D. Heinecke, and S. A. Diddams, Opt. Lett. 33, 1905 (2008).

13. T. Sakamoto, T. Kawanishi, and M. Tsuchiya, Opt. Lett. 33, 890 (2008).

14. I. Morohashi, T. Sakamoto, N. Sekine, A. Kasamatsu, and I. Hosako, Nano Commun. Netw. 10, 79 (2016).

15. I. Morohashi, M. Kirigaya, Y. Kaneko, I. Katayama, T. Sakamoto, N. Sekine, A. Kasamatsu, and I. Hosako, Proc. SPIE 9747, 97470U (2016). 